\title{
ANÁLISE DO EMPREGO DO GPR PARA ESTIMAR O TEOR DE UMIDADE DO SOLO A PARTIR DE UM ESTUDO NA CIDADE DE SÃO PAULO
}

\author{
Manuelle Santos Góis Paixão ${ }^{1}$, Renato Luiz Prado² e Liliana Alcazar Diogo ${ }^{3}$ \\ Recebido em 26 outubro, 2005 / Aceito em 30 junho, 2006 \\ Received on October 26, 2005 / Accepted on June 30, 2006
}

\begin{abstract}
This paper shows the results of soil moisture content measurements by GPR tests in a study area in São Paulo City, Brazil and discuss some aspects related to the accuracy of them.

Two different data interpretation methods were used: i) lineal adjustment of the arrival times of ground wave for different antenna separation; ii) time differences between the air and ground wave arrival times at a single antenna separation.

We used the RAMAC Mala Geosciences system with $50 \mathrm{MHz}, 100 \mathrm{MHz}$ and $200 \mathrm{MHz}$ antennas. The GPR survey was constituted of several multi-offset acquisition, using the WARR (Wide Angle Reflection and Refraction) geometry along of the profile in three different time periods (dry, intermediate and wet seasons). The distance increment between each WARR arrangement and traces was $0.1 \mathrm{~m}$ for the $100 \mathrm{MHz}$ and $200 \mathrm{MHz}$ antennas and $0.2 \mathrm{~m}$ for the $50 \mathrm{MHz}$.

At the same time of each GPR survey, soil samples, from $0.5 \mathrm{~m}$ up to $4 \mathrm{~m}$ depth, were collected, in $0.5 \mathrm{~m}$ intervals, for water content analysis in laboratory tests. The GPR data showed a good correlation with the laboratory data, but the results from the $200 \mathrm{MHz}$ antennas were the best one. The two data interpretation methods showed different accuracies. The time differences method was less precise due to the time shift in the ground wave arrival time at zero antenna offset that occurred in this survey. These results showed that it is very important to apply a correction factor for this effect, when it is present.
\end{abstract}

Keywords: GPR, moisture content, soil.

RESUMO. Este trabalho apresenta resultados de estudos visando à estimativa dos teores de umidade de solos superficiais com GPR em uma área teste na cidade de São Paulo/SP, Brasil, e à análise da acurácia dos processos envolvidos.

Foram empregadas duas metodologias diferentes: i) ajuste linear dos tempos de chegada da onda direta no solo para diferentes afastamentos; ii) diferenças dos tempos de chegada da onda aérea e da onda direta no solo para um único afastamento.

Utilizamos o equipamento da RAMAC Mala Geosciences com antenas de $50 \mathrm{MHz}, 100 \mathrm{MHz}$ e $200 \mathrm{MHz}$. A aquisição constituiu-se de diversos perfis multi-offset (múltiplos afastamentos) com o emprego da geometria WARR (Wide Angle Reflection and Refraction) ao longo de um perfil em três períodos climáticos distintos (seco, intermediário e úmido). Sendo o incremento entre os traços dos perfis multi-offset 0,2 m para a antena de $50 \mathrm{MHz}$ e 0,1 m para as antenas de $100 \mathrm{MHz}$ e $200 \mathrm{MHz}$. Concomitantemente ao levantamento GPR foram coletadas amostras de solo a intervalos de $0,5 \mathrm{~m}$ desde a superfície do terreno até a profundidade aproximada de 4,0 m para a determinação de seus teores de umidade em laboratório.

Os resultados do GPR apresentaram boa correlação com os dados obtidos através das análises de laboratório, sendo que os obtidos com as antenas de freqüência central de $200 \mathrm{MHz}$ propiciaram os melhores resultados. Os dois métodos empregados apresentaram diferentes acurácias. 0 método da diferença dos tempos de chegada da onda aérea e da onda direta no solo foi o menos preciso devido à ocorrência de um deslocamento de tempo da reta de ajuste dos tempos de chegada da onda direta no solo no afastamento zero. Esses resultados mostraram que, nesses casos, é importante aplicar um fator de correção para melhorar a acurácia dos resultados finais.

Palavras-chave: GPR, teor de umidade, solos.

\footnotetext{
${ }_{1}^{1}$ Programa de Pós-graduação, Instituto de Astronomia, Geofísica e Ciências Atmostéricas, Universidade de São Paulo, Rua do Matão, 1226, 05508-090 Cidade Universitária, São Paulo, SP, Brasil. Fax: (11) 3091-5034; Fone: (11) 3091-2792 - E-mail: manuelle@iag.usp.br

2 Instituto de Astronomia, Geofísica e Ciências Atmosféricas, Universidade de São Paulo, Rua do Matão, 1226, 05508-090 Cidade Universitária, São Paulo, SP, Brasil. Fax: (11) 3091-5034; Fone: (11) 3091-2762 - renato@iag.usp.br

${ }^{3}$ Instituto de Astronomia, Geofísica e Ciências Atmosféricas, Universidade de São Paulo, Rua do Matão, 1226 05508-090 Cidade Universitária, São Paulo, SP, Brasil. Fax: (11) 3091-5034; Fone: (11) 3091-4672 - liliana@iag.usp.br
} 


\section{INTRODUÇÃo}

A determinação de parâmetros hidrogeológicos, como nível d'água e teor de umidade do solo, tem uma ampla aplicação em estudos geoambientais (migração de contaminantes, balanceamento hídrico, exploração e contaminação de aqüíferos, etc.).

Uma vez que as propriedades eletromagnéticas na subsuperfície rasa são principalmente controladas pelo conteúdo de água (Topp et al., 1980), assim como as variações no tempo e espaço dessas propriedades podem estar associadas às mudanças do conteúdo de água, o emprego de métodos eletromagnéticos em estudos hidrogeológicos assume grande importância.

Na banda de freqüências empregada pelo método GPR (Ground Penetrating Radar), a velocidade de propagação da onda eletromagnética (EM) é principalmente determinada pela constante dielétrica, $K$, a qual possui valores próximos a 80 para a água; entre 3 e 10 para os principais minerais constituintes do solo, e 1 para 0 ar (Annan, 1998).

Dado 0 grande contraste entre essas constantes dielétricas, 0 método GPR permite a estimativa da distribuição do conteúdo de água em subsuperfície.

Para estimar o teor de umidade volumétrico dos solos com 0 GPR é necessário determinar a constante dielétrica a partir das leituras nos radargramas dos tempos de chegada da onda aérea e da onda direta no solo. No caso de um solo homogêneo, a parte da energia radiada que se propaga, diretamente, entre a antena transmissora e a receptora descreve uma relação linear entre 0 tempo de percurso e a distância entre as antenas, permitindo a determinação da velocidade de propagação da onda direta no solo e, conseqüentemente, a constante dielétrica, pela relação:

$$
V_{O D} \approx \frac{c}{\sqrt{K}}
$$

onde: $c=$ velocidade da luz $(0,3 \mathrm{~m} / \mathrm{ns})$ e $K=$ parte real da permissividade dielétrica ou constante dielétrica.

É importante observar que 0 uso da Eq. (1) é controverso para materiais argilosos. Olhoeft \& Capron (1994) indicam seu emprego somente para altas freqüências (acima de $300 \mathrm{MHz}$ ), condição para que a velocidade de propagação da onda eletromagnética não dependa da freqüência da onda. Contrariamente, White \& Zegelin (1995), baseando-se em resultados experimentais, assinalam que tal dependência não é significativa para freqüências maiores que $50 \mathrm{MHz}$, mesmo em solos argilosos.

Em vista disso, neste trabalho foram analisados os dados adquiridos com antenas de $50 \mathrm{MHz}, 100 \mathrm{MHz}$ e $200 \mathrm{MHz}$.

Para a estimativa dos teores de umidade foi empregada a proposição de Topp et al. (1980), a qual estabelece uma relação empírica entre a constante dielétrica $K$ e 0 teor de umidade à base de volume, $\theta\left(\mathrm{m}^{3} \cdot \mathrm{m}^{-3}\right)$, baseada em dados experimentais de laboratório. Seus dados foram obtidos a partir de amostras de sedimentos com diferentes composiçõos granulométricas e com 0 emprego de ondas eletromagnéticas com freqüências entre $1 \mathrm{MHz}$ e $1 \mathrm{GHz}$. A dependência entre esses dois parâmetros foi expressa matematicamente como:

$$
\begin{aligned}
\theta= & -5,3 \times 10^{-2}+2,92 \times 10^{-2} K \\
& -5,5 \times 10^{-4} K^{2}+4,3 \times 10^{-6} K^{3}
\end{aligned}
$$

\section{MÉTODOS}

Neste trabalho foi empregada a técnica multi-offset (levantamento WARR - Wide Angle Reflection and Refraction) em uma área de estudos hidrogeológicos no campus da Universidade de São Paulo - USP, na cidade de São Paulo/SP. 0 terreno é típico de áreas tropicais, ou seja, as frações de argila e silte contribuem de forma significativa na composição dos solos, tornando-os mais condutivos e, portanto, menos favorável ao emprego do GPR.

Há diversos relatos na literatura sobre o uso do GPR e o emprego da onda direta no solo para estimar o teor de umidade volumétrico (Du \& Rummel, 1994; Greaves et al.,1996; Van Overmeeren et al., 1997; Huisman et al., 2001; Garambois et al., 2002; Hubbard et al., 2002; Redman et al., 2003; Grote et al., 2003; Galagedara \& Parkin, 2003; Clement \& Ward, 2003; Huisman \& Bouten, 2003; Huisman et al., 2003).

Muitos destes autores empregaram, na aquisição, a geometria do afastamento constante (common offset) em situações de solos homogêneos de altas resistividades e obtiveram as constantes dielétricas a partir das diferenças dos tempos de chegada das ondas aérea e direta no solo, ou simplesmente por meio da análise qualitativa das variações dos tempos de chegada da onda direta no solo.

Neste estudo, para a estimativa dos teores de umidade foram testadas duas metodologias distintas: i) ajuste linear dos tempos de chegada da onda direta no solo para diferentes afastamentos e ii) diferenças dos tempos de chegada da onda aérea e da onda direta no solo, para um afastamento fixo adotado.

As antenas utilizadas foram de $50 \mathrm{MHz}, 100 \mathrm{MHz}$ e $200 \mathrm{MHz}$ (freqüências centrais) com incremento entre os traços dos perfis multi-offset de 0,2 m para a antena de $50 \mathrm{MHz}$ e $0,1 \mathrm{~m}$ para as antenas de $100 \mathrm{MHz}$ e $200 \mathrm{MHz}$, em diferentes períodos pluviométricos (janeiro-período chuvoso, abril-período intermediário e setembro-período seco) durante 0 ano de 2004, quando foi possível se observar uma acentuada variação da umidade entre os períodos chuvoso e seco. 


\section{Ajuste linear dos tempos de chegada da onda direta no solo}

Este método exige a identificação e leitura dos tempos de chegada da onda direta no solo, para os diferentes traços de cada conjunto multi-offset, e posterior ajuste linear destes dados no domínio tempo-distância. Dos coeficientes angulares obtêm-se os valores das velocidades para cada posição do arranjo, e a partir destes calculam-se as constantes dielétricas e os teores de umidade utilizando-se as Eqs. (1) e (2).

\section{Diferenças dos tempos da onda aérea $\left(t_{\mathrm{OA}}\right)$ e da onda direta no solo $\left(t_{\text {OD }}\right)$ para um afastamento específico}

Neste método, a constante dielétrica pode ser calculada a partir da Eq. (3), assumindo-se que as ondas aérea e direta chegam simultaneamente quando 0 afastamento entre as antenas é zero (Fig. 1a).

$$
K=\left(\frac{c\left(t_{O D}-t_{O A}\right)+x}{x}\right)^{2}
$$

onde: $t_{O D}=$ tempo da onda direta no solo; $t_{O A}=$ tempo da onda aérea e $x=$ afastamento.

Porém, Sperl (1999) apud Huisman \& Bouten (2003) relata que pode ocorrer em alguns levantamentos (como foi o caso dos ensaios realizados nesta pesquisa) uma diferença de tempo entre as chegadas dessas ondas no afastamento zero. A origem dessa diferença, embora ainda não totalmente compreendida, é atribuída às características de radiação das antenas do GPR e/ou distorções das formas dos pulsos das ondas aérea e direta no solo devido ao imperfeito acoplamento elétrico das antenas (Huisman \& Bouten, 2003). Assim Sperl sugere a inclusão de um fator de correção, $t_{0}$ (Fig. 1b), na Eq. (3) tornando-a:

$$
K=\left(\frac{c\left(t_{O D}-t_{0}-t_{O A}\right)+x}{x}\right)^{2}
$$

onde: $t_{0}=$ diferença de tempo entre as chegadas das ondas direta no solo e aérea observada em $x$ igual a zero.

Com a Eq. (5) obtém-se a velocidade da onda direta no solo $\left(V_{O D}\right)$ por meio dos tempos de chegada das ondas aérea $\left(t_{O A}\right)$ e direta no solo $\left(t_{O D}\right)$ para 0 afastamento $(x)$ e da velocidade da onda aérea $\left(V_{O A}\right)$.

$$
V_{O D}=\frac{1}{\left[\frac{t_{O D}-t_{O A}-t_{0}}{x}+\frac{1}{V_{O A}}\right]}
$$

Observando-se a ocorrência de diferença de tempo $t_{0}$ nos radargramas, o emprego da Eq. (5) proporcionará uma maior acurácia às determinações dos teores de umidade, como observado mais adiante.

\section{Aspectos relacionados à acurácia do método}

A acurácia da estimativa do teor de umidade volumétrico depende fundamentalmente da correta determinação do tempo de chegada da onda direta no solo. Observou-se neste estudo que a leitura deste evento nos registros obtidos com as antenas de freqüência central mais baixa, e.g. $50 \mathrm{MHz}$ e $100 \mathrm{MHz}$, mostrou-se muito difícil, principalmente nos períodos mais secos (baixo índice pluviométrico), gerando imprecisão na determinação da constante dielétrica e, conseqüentemente, no teor de umidade.

A dificuldade na identificação e leitura correta do tempo de chegada da onda direta no solo advém da superposição de fases dos eventos relacionados à refração crítica na interface solo/ar e às oscilações da onda aérea - ringing da onda aérea. Observouse que as oscilações das ondas aéreas foram mais pronunciadas nos períodos secos, principalmente nos registros feitos com as antenas de $50 \mathrm{MHz}$ e $100 \mathrm{MHz}$ como ilustram as Figs. 2d, 2e e 2f.

Esse problema é minimizado quanto maior for 0 afastamento entre as antenas. Nas Figs. 2d, 2e e $2 f$ nota-se claramente que a identificação do tempo de chegada da onda direta no solo para as antenas de baixas freqüências, tanto no período seco quanto no chuvoso, é dificultada pela interferência de fases da onda área.

Já o radargrama da antena de $200 \mathrm{MHz}$ em janeiro (período chuvoso) possibilitou a identificação precisa dos eventos da onda aérea e da onda direta no solo (Fig. 2a e Fig. 3). Pode-se observar uma clara distinção entre os coeficientes angulares das velocidades de fase da onda área e onda direta no solo (Fig. 3).

\section{RESULTADOS}

\section{Umidade natural do solo}

Para os ensaios de teor de umidade natural as amostras foram coletadas na área de estudos com um trado mecânico a intervalos de $0,5 \mathrm{~m}$ desde a superfície do terreno até a profundidade aproximada de 4,0 $\mathrm{m}$ (referente ao nível freático local). Essas amostras foram colocadas em recipientes de massa conhecida e lacradas para não perder 0 teor de umidade original até a realização dos ensaios de laboratório.

Para determinar 0 teor de umidade natural do solo de cada horizonte foram medidas as massas das amostras e colocadas numa estufa de ventilação forçada a $105^{\circ} \mathrm{C}$ por $24 \mathrm{~h}$; em seguida foram medidas as massas das amostras secas, sendo a diferença da massa úmida das amostras menos a massa seca dividida pela massa seca 0 teor de umidade natural de cada horizonte (Fig. 4).

A análise granulométrica das amostras revela um horizonte superficial arenoso com granodecrescência com 0 aumento da profundidade até constituir um horizonte de argila entre 1,2 $\mathrm{m}$ e 2,0 m. A partir da base da camada de argila ocorre nova transição 

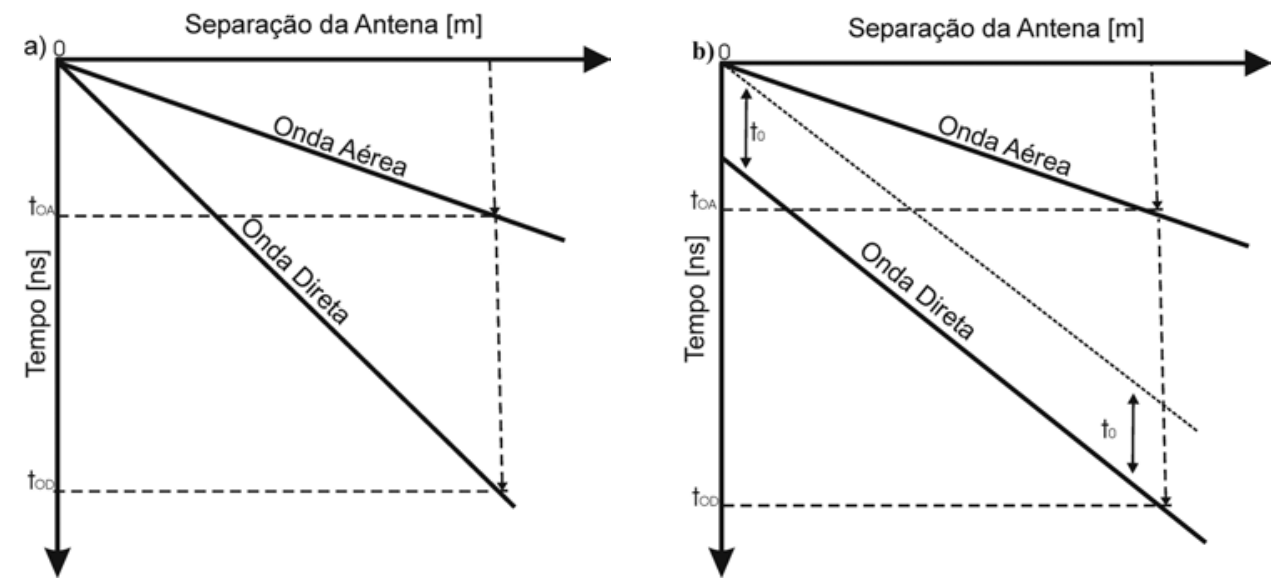

Figura 1 - Gráficos esquemáticos dos tempos de chegada dos diversos eventos em função do afastamento em um levantamento multi-offset: a) onda direta com origem no afastamento zero; b) onda direta deslocada de um $t_{0}$ em relação à origem (modificado de Huisman \& Bouten, 2003).

para sedimentos arenosos até o nível freático em 4,0 m de profundidade.

0 horizonte correspondente ao pacote de argilas tem o maior teor de umidade, que diminui com a profundidade à medida que os sedimentos tornam-se predominantemente arenosos.

\section{Ajuste linear}

As Figs. 5, 6 e 7 apresentam os teores de umidade obtidos pelo método do ajuste linear dos tempos de chegada da onda direta no solo, extraídos dos conjuntos multi-offset para os diferentes períodos pluviométricos e diferentes antenas.

Os índices pluviométricos para os ensaios nos períodos de janeiro, abril e setembro, foram respectivamente $203 \mathrm{~mm}, 80 \mathrm{~mm}$ e $19 \mathrm{~mm}$ (IAG, 2002). Para os três períodos, os teores de umidade estimados para cada antena seguiram, em média, a mesma tendência $\theta_{200 \mathrm{MHz}}>\theta_{100 \mathrm{MHz}}>\theta_{50 \mathrm{MHz}}$.

Para o período seco os teores de umidade são menores quando comparados com os outros períodos mantendo em média a mesma tendência $\theta_{200 \mathrm{MHz}}>\theta_{100 \mathrm{MHz}}>\theta_{50 \mathrm{MHz}}$. Há desvios nessa tendência em alguns pontos do perfil, atribuídos à comentada imprecisão na determinação dos tempos de chegada da onda direta no solo nos radargramas.

Os teores obtidos pelo método do ajuste linear são mais próximos daqueles observados nas amostras do horizonte de solo mais superficial $(<0,5 \mathrm{~m})$. Portanto, independente da freqüência da antena utilizada, o método empregado permite apenas a investigação dos materiais mais superficiais do terreno.

Os resultados pelo método do ajuste linear, obtidos com as antenas de $100 \mathrm{MHz}$ e $200 \mathrm{MHz}$, são bem coerentes com as variações sazonais de umidade observadas nas amostras de solo, porém os obtidos com as antenas de $50 \mathrm{MHz}$ apresentam valores inferiores, provavelmente devido às interferências das oscilações da onda aérea discutidas anteriormente.

\section{Diferença $\mathbf{t}_{\mathbf{O D}}-\mathbf{t}_{\mathbf{O A}}$}

A metodologia foi empregada somente para os dados adquiridos com a antena de $200 \mathrm{MHz}$, pois os eventos relativos às chegadas das ondas aérea e direta no solo puderam ser melhor distinguidos nos radargramas, fato que não se observou nos registros obtidos com as antenas de $100 \mathrm{MHz}$ e $50 \mathrm{MHz}$, como já comentado.

Foram examinados diferentes valores de afastamento e escoIhidos os de 1,0 m, 2,0 m e 2,8 m para observar-se como as interferências das oscilações da onda aérea (ringing) podem afetar os valores finais de umidade obtidos.

Para o cálculo de $K$ utilizou-se a Eq. (4). 0 resultado obtido com 0 afastamento de 2,8 m, no qual ocorre a menor interferência do ringing da onda aérea, foi o que mais se aproximou do obtido com o método do ajuste linear para diferentes afastamentos (Fig. 8).

A Figura 9 apresenta os resultados que seriam obtidos se 0 valor de $t_{0}$ fosse desconsiderado. Neste caso, 0 ajuste linear busca uma reta que passe pela origem (afastamento $0 \mathrm{~m}$ ), 0 que de fato não ocorre com os dados adquiridos, gerando falsas estimativas dos teores de umidade.

No caso estudado fica bem claro que a desconsideração do fator de correção induziria a grandes erros no cálculo final dos teores de umidade.

Outra observação importante feita nesses ensaios é que o fator de correção, $t_{0}$, variou ao longo do perfil realizado. Essas variações se devem às imprecisões nas leituras dos tempos de 

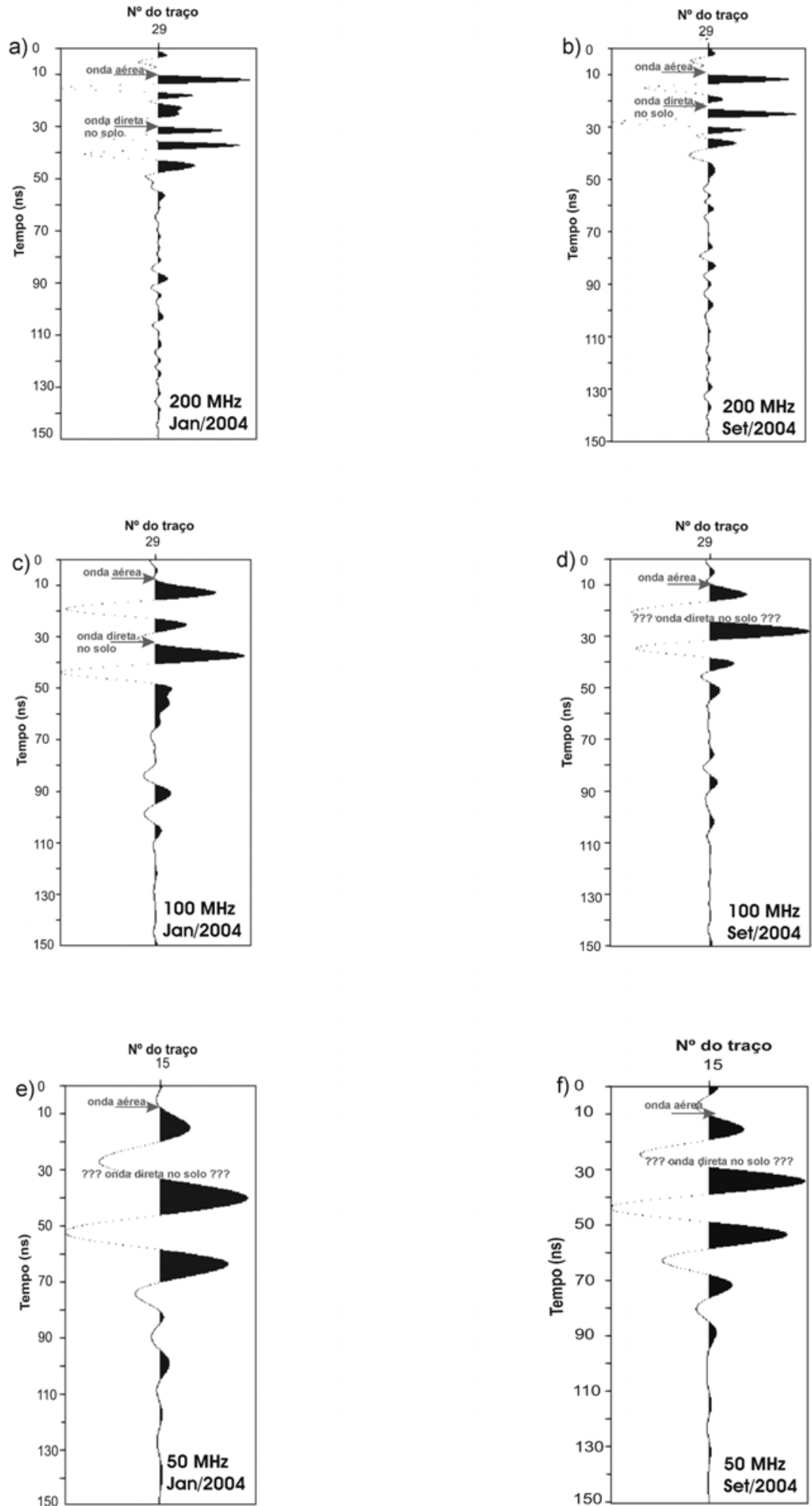

Figura 2 - Traços de GPR para diferentes antenas a) $200 \mathrm{MHz}$ janeiro/2004 (período chuvoso); b) 200 MHz setembro/2004 (período seco - onda direta identificada em 'a' e 'b'); c) $100 \mathrm{MHz}$ janeiro/2004 (período chuvoso - onda direta identificada); d) 100 MHz setembro/2004 (período seco); e) $50 \mathrm{MHz}$ janeiro/2004 (período chuvoso) e f) $50 \mathrm{MHz}$ setembro/2004 (período seco - difícil identificação da onda direta em 'd', 'e', e 'f'). 


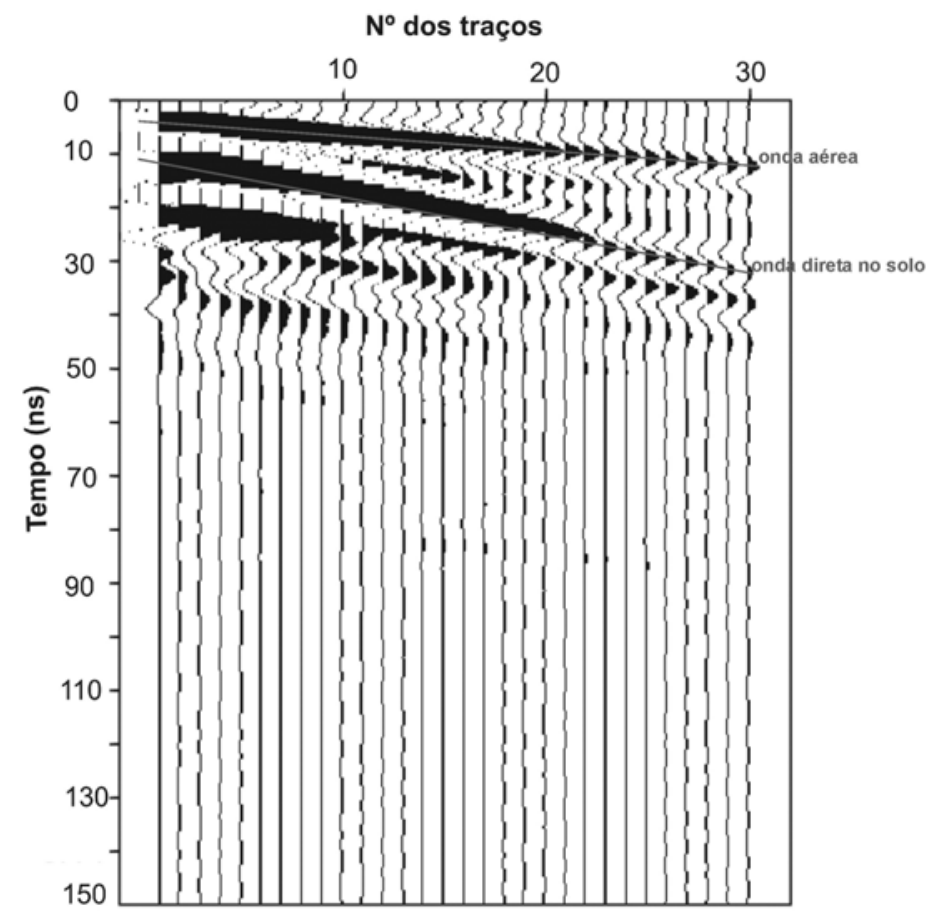

a)

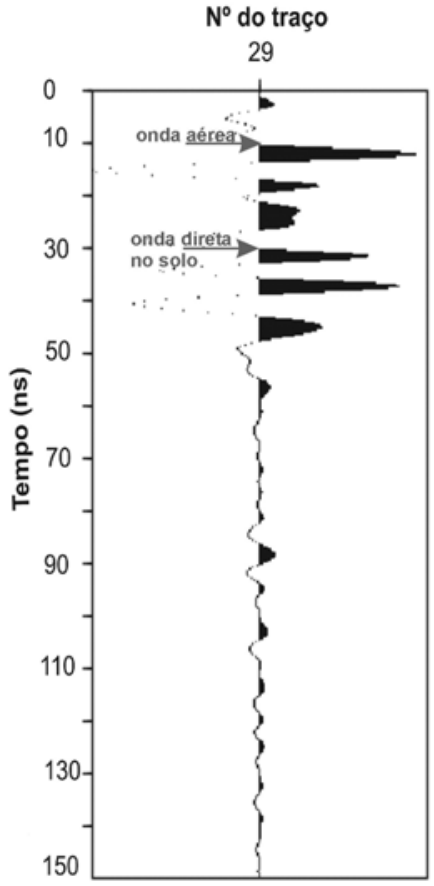

b)

Figura 3 - a) Levantamento multi-offset de $200 \mathrm{MHz}$ obtido em janeiro/2004 (período chuvoso) com a onda aérea e a onda direta e b) traço 29 do mesmo registro evidenciando o início e término da onda direta e da onda aérea (mesmo traço da Figura 2a).

Teor de umidade

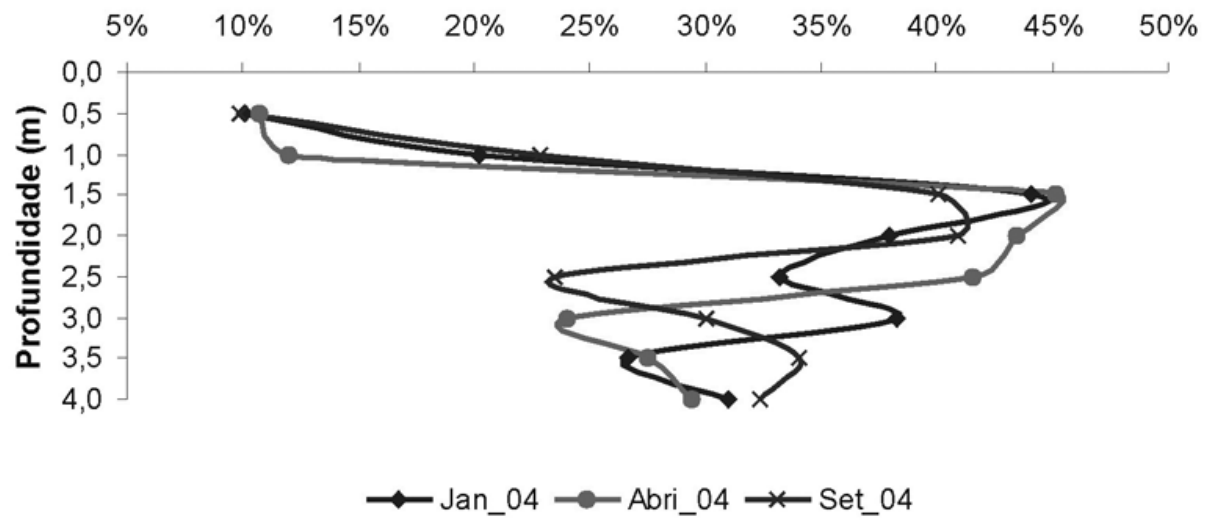

Figura 4 - Perfis dos teores de umidade natural medido em amostras de solo da área de estudos para todas as campanhas: janeiro/2004 (período chuvoso), abril/2004 (período intermediário) e setembro/2004 (período seco).

chegada, erros de posicionamento, variações nas condições do acoplamento antena/solo (Fig. 10). A variação observada, embora possa parecer pequena (média próxima de $8 \mathrm{~ns}$ ), acarreta erros significativos nos valores de umidade.

Portanto, a adoção do método da diferença $\left(t_{O D}-t_{O A}\right)$ em um levantamento GPR padrão, do tipo common offset exige a execução de, pelo menos, um levantamento multi-offset, e neste caso com a presunção de que $t_{0}$ será constante ao longo de todo o levantamento, o que pode não ser rigorosamente correto, como mostra a Fig. 10. 


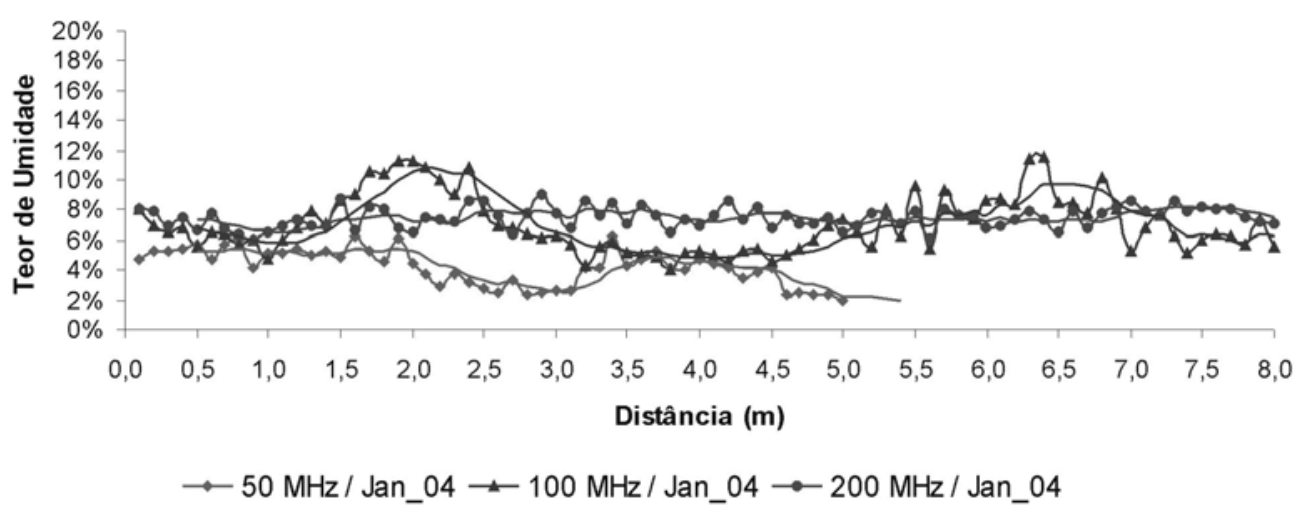

Figura 5 - Estimativa dos valores do teor de umidade na campanha de janeiro/2004 (período chuvoso) para as antenas de $50 \mathrm{MHz}, 100 \mathrm{MHz}$ e $200 \mathrm{MHz}$ de freqüências centrais. A linha cheia representa média móvel de período 5 para cada conjunto de dados.

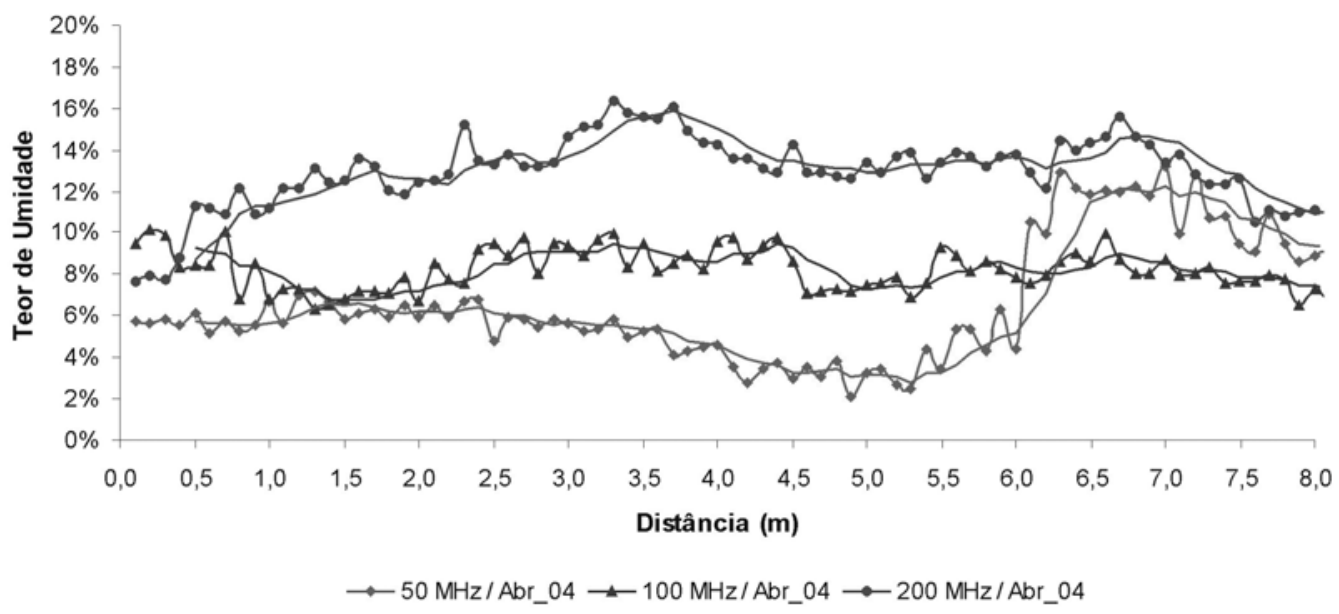

Figura 6 - Estimativa dos valores do teor de umidade na campanha de abril/2004 (período intermediário) para as antenas de $50 \mathrm{MHz}, 100 \mathrm{MHz}$ e $200 \mathrm{MHz}$ de frequêencias centrais. A linha cheia representa média móvel de período 5 para cada conjunto de dados.

\section{CONCLUSÕES}

As duas metodologias foram sensíveis às variações de umidade entre os períodos com diferentes índices pluviométricos, principalmente entre os períodos chuvoso e seco.

A estimativa do teor de umidade volumétrico utilizando 0 ajuste linear para diferentes afastamentos apresentou valores bem próximos aos obtidos com a medida direta, que variaram, aproximadamente, de $6,5 \%$ a $10 \%$ para os horizontes mais rasos $(<0,50 \mathrm{~m})$.

Pelo método das diferenças, para um afastamento de 2,8 m, a antena de $200 \mathrm{MHz}$ foi a que apresentou maior acurácia na estimativa do teor de umidade volumétrico, visto que os eventos da onda aérea e direta mostraram-se, neste caso, bem diferenciados nos radargramas.

Aplicando-se o método das diferenças é fundamental investi- gar a ocorrência ou não do deslocamento do tempo da onda direta $\left(t_{0}\right)$, pois a acurácia do método depende desta informação.

Os resultados indicaram que as estimativas dos teores de umidade obtidos por ambos os métodos utilizados nesse trabalho corresponderam aos dos horizontes mais rasos (até 0,5 m de profundidade, aproximadamente), independentemente da freqüência central da antena empregada.

\section{AGRADECIMENTOS}

À Coordenação de Aperfeiçoamento de Pessoal de Nível Superior - CAPES; Ao Instituto de Astronomia Geofísica e Ciências Atmosféricas - IAG/USP - Departamento de Geofísica e à Fundação de Amparo à Pesquisa do Estado de São Paulo - FAPESP (auxílio pesquisa 2003/08903-8). 


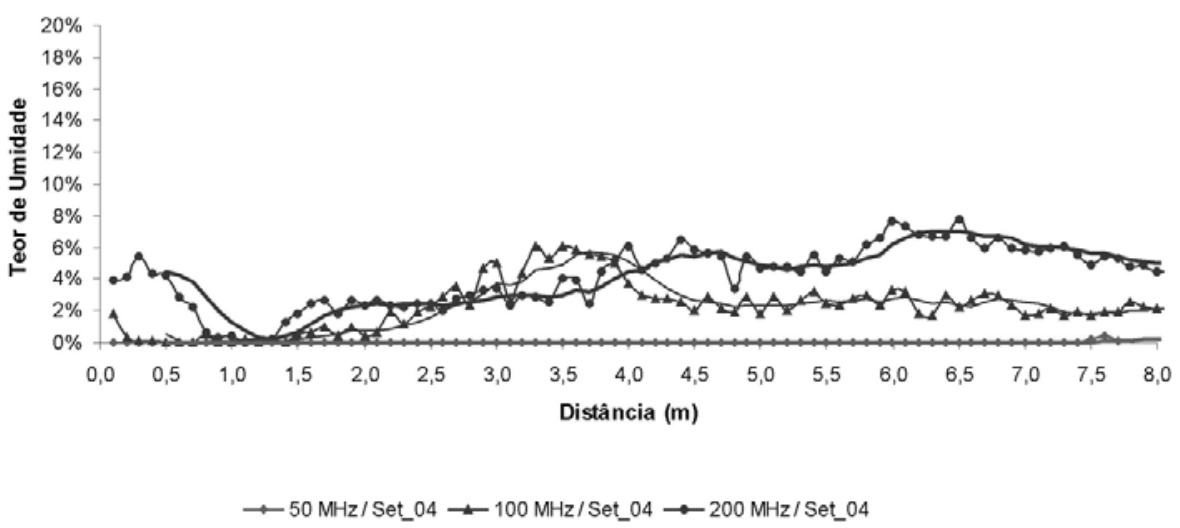

Figura 7 - Estimativas dos valores do teor de umidade na campanha de setembro/2004 (período seco) para as antenas de $50 \mathrm{MHz}, 100 \mathrm{MHz}$ e $200 \mathrm{MHz}$ de freqüências centrais. A linha cheia representa média móvel de período 5 para cada conjunto de dados.

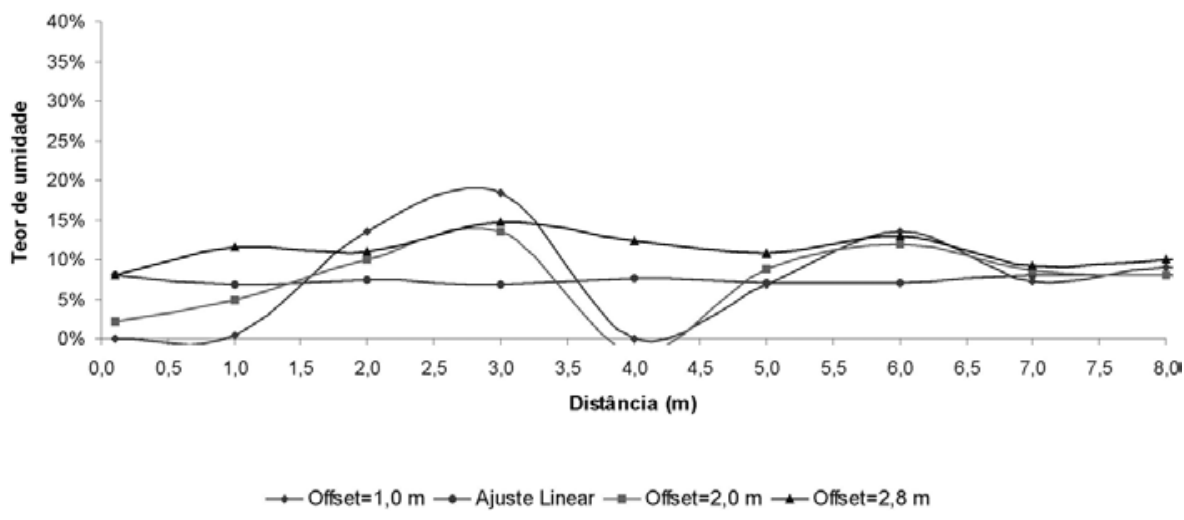

Figura 8 - Estimativa dos valores do teor de umidade na campanha de janeiro/2004 (período chuvoso) para os diferentes offsets com a antena de $200 \mathrm{MHz}$ de freqüência central considerando 0 deslocamento $t_{0}$.

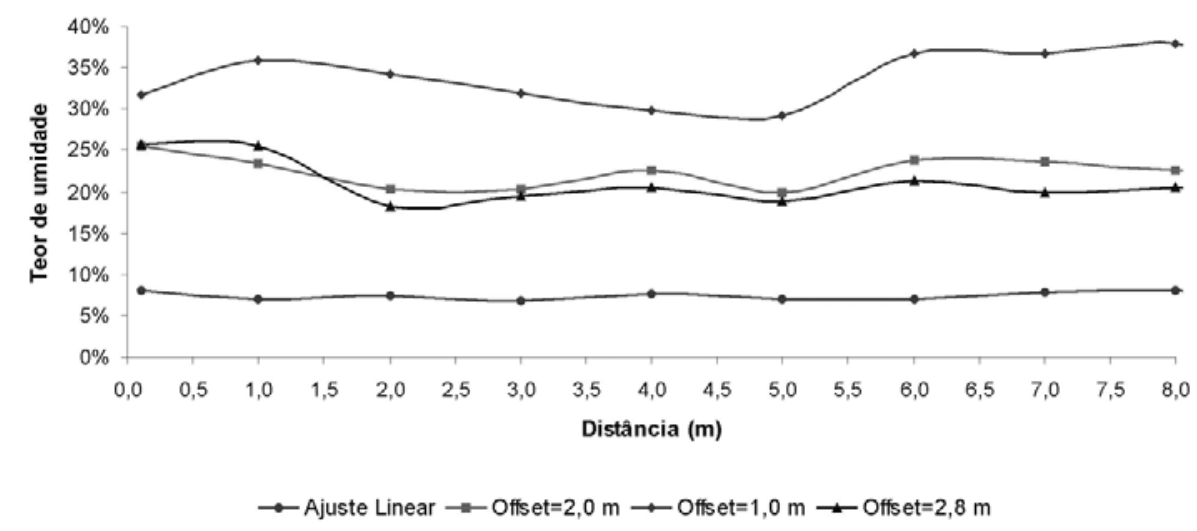

Figura 9 - Estimativa dos valores do teor de umidade na campanha de janeiro/2004 (período chuvoso) para os diferentes afastamentos com a antena de $200 \mathrm{MHz}$ de freqüência central sem considerar o deslocamento $t_{0}$ 


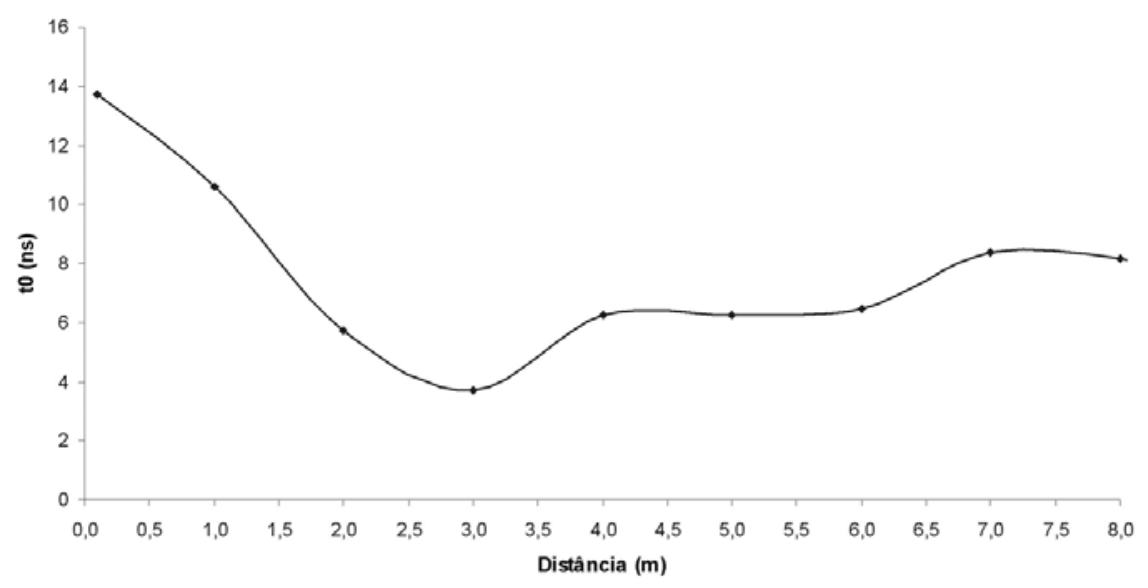

Figura 10 - Valores do deslocamento $t_{0}$ para os diferentes pontos da antena transmissora na campanha de janeiro/2004 (período chuvoso) para 0 offset $x=2,8 \mathrm{~m}$ com a antena de $200 \mathrm{MHz}$ de freqüência central.

\section{REFERÊNCIAS}

ANNAN AP. 1998. Ground Penetrating Radar: Workshop Notes. Canada: Sensors \& Software Inc., $33 p$.

CLEMENT WP \& WARD AL. 2003. Using Ground Penetrating Radar to Measure Soil Moisture Content. American Society of Agricultural Engineering-ASAE. In: Annual Meeting, paper number 032275, $14 \mathrm{p}$.

DU S \& RUMMEL P. 1994. Reconnaissance Studies of Moisture in the Subsurface with GPR, In: Proceedings of The Fifth International Conference on Ground Penetrating Radar, GPR 94, p. 1241-1248.

GALAGEDARA LW \& PARKIN GW. 2003. An Analysis of the GPR Direct Ground Wave Method for Soil Water Content Measurement. CGRG, Hydrological Processes, p. 3615-3628.

GARAMBOIS S, SÉNÉCHAL P \& PERROUD H. 2002. On the use of combined geophysical methods to assess water content and water conductivity of near-surface formations. Journal of Hydrology, 259: 32-48.

GREAVES RJ, LESMES DP, LEE MJ \& TOKSÖZ NM. 1996. Velocity variations and water content estimated from multi-offset, ground-penetrating radar. Geophysics, 61: 683-695.

GROTE K, HUBBARD SS \& RUBIN Y. 2003. Field-Scale Estimation of Volumetric Water Content using GPR Groundwave Techniques. Water Resources Research, 39 (11).

HUBBARD S, GROTE K \& RUBIN Y. 2002. Mapping the volumetric soil water content of a California vineyard using high-frequency GPR ground wave data. Leading Edge Explor. 21: 552-559.

HUISMAN JA, SPERL C, BOUTEN W \& VERSTRATEN JM. 2001. Soil water content measurements at different scales: accuracy of time domain reflectometry and ground-penetrating radar, Journal of Hydrology, 245: 48-58.
HUISMAN JA \& BOUTEN W. 2003. Accuracy and Reproducibility of Mapping Surface Soil Water Content with the Ground Wave of GroundPenetrating Radar. Journal of Environmental and Engineering Geophysics. 8: 67-75.

HUISMAN JA, HUBBARD SS, REDMAN JD \& ANNAN AP. 2003. Measuring Soil Water Content with Ground Penetrating Radar: A Review. Vadose Zone Journal. 2: 476-491.

IAG. 2002. Observações de Superfície Efetuadas na Estação Meteorológica. Seção Técnica de Serviços Meteorológicos - Instituto de Astronomia, Geofísica e Ciências Atmosféricas, Universidade de São Paulo. v. 6.

OLHOEFT GR \& CAPRON DE. 1994. Petrophysical causes of electromagnetic dispersion: in Proc. of the Fifth Int'I. Conf. on Ground Penetrating Radar, Kitchener, Ontario, 12-16 June, p. 145-152.

REDMAN D, GALAGEDARA L \& PARKIN G. 2003. Measuring Soil Water Content with the Ground Penetrating Radar Surface Reflectivity Method: Effects of Spatial Variability. In: ASAE Annual International Meeting Sponsored.

TOPP GC, DAVIS JL \& ANNAN AP. 1980. Electromagnetic Determination of Soil Water Content: Measurements in Coaxial Transmission Lines. Water Resources Research, 16: 574-582.

VAN OVERMEEREN RA, SARIOWAN SV \& GEHRELS JC. 1997. Ground penetrating radar for determining volumetric soil water content; results of comparative measurements at two test sites. Journal of Hydrology, p. 316-338.

WHITE I \& ZEGELIN SJ. 1995. Electric and dielectric methods for monitoring soil-water content. In: WILSON LG, EVERETT LG \& CULLEN SJ (Ed.). Handbook of Vadose Zone Characterization and Monitoring: principles, Methods and Case Studies. Lewis Publishers: Boca Raton, Florida, p. 343-385. 


\section{NOTAS SOBRE OS AUTORES}

Manuelle Santos Góis Paixão. Bacharel em Física pela Universidade Estadual de Santa Cruz (UESC-BA) em 2003, mestre em Geofísica pelo Instituto de Astronomia, Geofísica e Ciências Atmosféricas (IAG-USP) em 2005 e atualmente doutoranda em Geofísica pelo IAG-USP.

Renato Luiz Prado. Bacharel em Geologia (IGc/USP, 1981), mestre em Geofísica (IAG/USP, 1994) e doutor em Geociências e Meio Ambiente (IGCE/UNESP, 2000). De 1982 a 2001 foi pesquisador no Laboratório de Geofísica Aplicada do Instituto de Pesquisas Tecnológicas - IPT, São Paulo. Desde 2001 é professor no Departamento de Geofísica do IAG/USP. Áreas de interesse: métodos sísmicos e GPR.

Liliana Alcazar Diogo. Graduada em Geofísica em 1989 pela Universidade de São Paulo (IAG/USP). Doutora em Geofísica em 1995 pela Universidade Federal da Bahia (PPPG/UFBA). Docente do Departamento de Geofísica do Instituto de Astronomia, Geofísica e Ciências Atmosféricas da Universidade de São Paulo, com atuação na área de Geofísica Aplicada, com ênfase em métodos sísmicos na escala de investigação rasa. 